Revista Mídia e Cotidiano

ISSN: 2178-602X

Volume 15, Número 1, jan./abr. de 2021

\title{
Expediente
}

\section{Equipe Ediitoriall}

\section{EDITORAS}

- Denise TAVARES, Universidade Federal Fluminense - UFF, Brasil

- Isabella REGA, Bournemouth University, Reino Unido

- Renata TOMAZ, Universidade Federal Fluminense - UFF, Brasil

\section{EDITORES ASSISTENTES}

- Davi REBOUÇAS - Universidade Federal Fluminense - UFF, Brasil

- Diego de Souza COTTA - Universidade Federal Fluminense - UFF, Brasil

- Pedro Henrique Conceição dos SANTOS - Universidade Federal Fluminense - UFF, Brasil

- Mateus QUEIROZ - Universidade Federal Fluminense - UFF, Brasil

\section{CAPA}

- Denise TAVARES, Universidade Federal Fluminense - UFF, Brasil (com projeto gráfico de Vitor Silva CHAGAS).

\section{Foto CAPA}

- Rennato Testa (Zaragoza, capital da região de Aragão, nordeste da Espanha).

\section{Consellho Científico}

- Aimée Vega-Montiel, Universidad Nacional Autónoma de México - UNAM, México

- Ana Carolina Rocha Pessôa Temer, Universidade Federal de Goiás - UFGO, Brasil

- Carlos Alberto Zanotti, Pontifícia Universidade Católica de Campinas - PUC-Campinas, Brasil

- Christina Ferraz Musse, Universidade Federal de Juiz de Fora - UFJF, Brasil

- Cristiane Freitas Gutfreind, Pontifícia Universidade Católica do Rio Grande do Sul, Brasil

- Clementina Galera Casquet, Universidad de Extremadura, Espanha

- Dênis de Moraes, Universidade Federal Fluminense - UFF, Brasil

- Denise da Costa Oliveira Siqueira, Universidade do Estado do Rio de Janeiro, Brasil

- Edson Dalmonte, Universidade Federal da Bahia - UFBA, Brasil 
- Francisco Karam, Universidade Federal de Santa Catarina - UFSC, Brasil

- Gabriela Borges Martins Caravela, Universidade Federal de Juiz de Fora, Brasil

- Gabriel Kaplún, Universidad de la República Uruguay, Uruguai

- Helena Sousa, Universidade do Minho, Portugal

- Joseph Straubhaar, University of Texas at Austin, Estados Unidos da América do Norte

- Katia Lerner, Fundação Oswaldo Cruz, Brasil

- Liriam Sponholz, Department of Media and Communication, Alemanha

- Lucília de S. Romão, Universidade de São Paulo - USP, Brasil

- Luis Albornoz, Universidad Carlos III de Madrid, Espanha

- Marcius Freire, Universidade Estadual de Campinas - UNICAMP, Brasil

- Marcos Palácio, Universidade da Beira Interior - UBI, Portugal

- Maria Aparecida Baccega, Escola Superior de Propaganda e Marketing - ESPM, Brasil

- Marta Martín Llaguno, Universidad de Alicante - UA, Espanha

- Massimo Canevacci, Università degli Studi di Roma La Sapienza, Itália

- Mauricio Ribeiro da Silva, Universidade Paulista - UNIP/SP, Brasil

- Muniz Sodré, Universidade Federal do Rio de Janeiro - UFRJ, Brasil

- Nelson Zagalo, Universidade de Aveiro, Portugal

- Nilda A. Jacks, Universidade Federal do Rio Grande do Sul - UFRGS, Brasil

- Nísia Martins do Rosário, Universidade Federal do Rio Grande do Sul - UFRS, Brasil

- Raquel Paiva, Universidade Federal do Rio de Janeiro - UFRJ, Brasil

- Salvatore Scifo, Bournemouth University, Reino Unido

- Sandra Fisher, Universidade Tuiuti do Paraná, Curitiba

- Tanja Dreher, University of New South Wales, Sydney, Austrália

Data da Publicação: 19 de janeiro de 2021

www.ppgmidiaecotidiano.uff.br www.periodicos.uff.br/midiaecotidiano midiaecotidiano.ega@id.uff.br 